\title{
Revisiting the Growth of Black Phosphorus in Sn-I Assisted Reactions
}

\author{
Dongya Wang ${ }^{1}$, Peng $\mathrm{Yi}^{1}{ }^{1}$, Lin Wang ${ }^{1}$, Lu Zhang ${ }^{1}$, Hai Li ${ }^{1}$, Min Lu ${ }^{1 *}$, Xiaoji Xie ${ }^{1 *}$, \\ Ling Huang ${ }^{1}$ and Wei Huang ${ }^{1,2}$ \\ ${ }^{1}$ Key Laboratory of Flexible Electronics (KLOFE), Institute of Advanced Materials, Nanjing Tech University, Nanjing, China, \\ ${ }^{2}$ Shaanxi Institute of Flexible Electronics, Northwestern Polytechnical University, Xi'an, China
}

OPEN ACCESS

Edited by:

Luís António Dias Carlos,

University of Aveiro, Portugal

Reviewed by:

Amir Pakdel,

Trinity College Dublin, Ireland

Zhong Jin,

Nanjing University, China

*Correspondence:

Min Lu

iamm/v@nitech.edu.cn

Xiaoji Xie

iamxjxie@njtech.edu.cn

Specialty section:

This article was submitted to Inorganic Chemistry,

a section of the journal

Frontiers in Chemistry

Received: 04 December 2018

Accepted: 10 January 2019

Published: 28 January 2019

Citation:

Wang D, Yi P, Wang L, Zhang L, Li H, $L u M$, Xie $X$, Huang $L$ and Huang $W$

(2019) Revisiting the Growth of Black

Phosphorus in Sn-l Assisted

Reactions. Front. Chem. 7:21.

doi: 10.3389/fchem.2019.00021
Black phosphorus, an emerging layered material, exhibits promising applications in diverse fields, ranging from electronics to optics. However, controlled synthesis of black phosphorus, particularly its few-layered counterparts, is still challenging, which should be due to the unclear growth mechanism of black phosphorus. Here, taking the most commonly used Sn-I assisted synthesis of black phosphorus as an example, we propose a growth mechanism of black phosphorus crystals by monitoring the reactions and analyzing the as-synthesized products. In the proposed mechanism, $\mathrm{Sn}_{24} \mathrm{P}_{19.3} \mathrm{l}_{8}$ is the active site for the growth of black phosphorus, and the black phosphorus crystals are formed with the assistance of $\mathrm{Snl}_{2}$, following a polymerization-like process. In addition, we suggest that all Sn-I assisted synthesis of black phosphorus should share the same reaction mechanism despite the differences among Sn-I containing additives. Our results shown here should shed light on the controlled synthesis of black phosphorus and facilitate further applications of black phosphorus.

Keywords: black phosphorus, growth mechanism, tin iodide, chemical vapor transport, 2D materials

\section{INTRODUCTION}

With the rapid development of two-dimensional materials, orthorhombic black phosphorus (BP), assembled by puckered phosphorus layers of interlinked six-membered rings via van der Waals interactions, recently has attracted much research enthusiasm due to its layer-number-dependent properties (Hirsch and Hauke, 2018; Liu H. et al., 2018; Liu Y. et al., 2018). Specifically, few-layered BP possesses tunable band gap, ranging from 0.3 to $2.0 \mathrm{eV}$ (Xia et al., 2014), and high carrier mobility $\left(\sim 1,000 \mathrm{~cm}^{2} \mathrm{~V}^{-1} \mathrm{~s}^{-1}\right)$ (Li et al., 2014), which makes it promising for diverse applications, including field effect transistor, battery, sensor, and electrocatalyst (Liu et al., 2015; Zhang Y. et al., 2017; Hu et al., 2018). Despite the outstanding properties of BP and the recent inspiring studies, the exploration of the properties of $\mathrm{BP}$ and corresponding applications are still constrained to some extent because of the difficulties in synthesizing BP and its layered counterparts (Hu et al., 2018; Zhang et al., 2018).

In fact, $\mathrm{BP}$ is a thermodynamically stable form of phosphorus but does not exist naturally. Formerly, BP was synthesized by transforming red phosphorus or white phosphorus under harsh conditions (Bridgman, 1914, 1935, 1948). It was until 2007 that a simple synthetic approach based on Sn-I assisted chemical vapor transport reactions was developed for producing BP crystals in high quality and high yield (Lange et al., 2007). In this method, BP is synthesized, in an evacuated ampoule, by programmed heating of red phosphorus with the 
mineralizers (e.g., $\mathrm{Sn} / \mathrm{SnI}_{4}$ ). It should be emphasized that the mineralizers, particularly Sn and I elements, are decisive in this chemical vapor transport based method. Currently, this Sn-I assisted method is the most commonly used strategy for the largescale preparation of high quality BP crystals, which indeed boosts both the scientific research and technological development of BP (Nilges et al., 2008; Köpf et al., 2014). Nevertheless, controlled synthesis of BP, particularly the synthesis of uniform thin BP film (few-layered BP), has not been achieved by chemical vapor transport based methods yet (Yang et al., 2015; Smith et al., 2016).

In order to realize controlled synthesis of $\mathrm{BP}$, it is crucial to fully understand the corresponding formation mechanisms. Unfortunately, the formation mechanisms of BP in the Sn-I assisted method are still unclear, although several efforts have been devoted to revealing the detailed formation processes (Zhao et al., 2016a,b; Li et al., 2017; Zhang Z. et al., 2017; Shriber et al., 2018). Furthermore, the currently proposed formation mechanisms are different from each other, although they share some opinions. For example, in 2016, a molten alloy based mechanism was proposed (Zhao et al., 2016a). In this mechanism, BP crystals were believed to precipitate from the molten alloy of red phosphorus and metallic Sn when the temperature decreased. At almost the same time, a phase-transfer mechanism was proposed, in which BP crystals were proposed to be transformed from Hittorf's phosphorus with the assistance of a certain PSn-I ternary compound (Zhang Z. et al., 2017). Later on, BP crystals were suggested to grow, obeying a vapor-solid-solid mechanism, through the diffusion of excess $\mathrm{P}$ atoms from a $\mathrm{Sn}_{24} \mathrm{P}_{22-\mathrm{x}} \mathrm{I}_{8}(\mathrm{x} \approx 2.7)$ intermediate compound (Li et al., 2017). In addition to the studies based on experimental observations, another BP formation pathway was advised recently according to first-principle calculations (Shriber et al., 2018). According to the density functional theory calculations, BP is favorably formed, in the presence of Sn-I containing mineralizer at high temperature and pressure, by a series of additions of $\mathrm{P}_{4}$ molecules in a polymerization-like process. Collectively, more efforts are desired to elucidate the formation mechanisms of BP crystals in the Sn-I assisted synthetic method.

In this study, taking the previous studies into account, we propose a new formation mechanism of BP crystals in the SnI assisted reaction according to a series of reaction observations and product characterization. In our proposed mechanism, the Sn and I containing mineralizers first decompose, forming $\mathrm{SnI}_{2}$ and $\mathrm{Sn}$, and then the decomposed compounds react with phosphorus vapor at elevated temperature to form $\mathrm{Sn}_{24} \mathrm{P}_{19.3} \mathrm{I}_{8}$. Subsequently, the $\mathrm{Sn}_{24} \mathrm{P}_{19.3} \mathrm{I}_{8}$ molecules transport to and partially deposit at the zone with slightly low temperature $\left(\sim 550-500^{\circ} \mathrm{C}\right)$ during the cooling stage, generating the growth sites for BP crystals. The $\mathrm{Sn}_{24} \mathrm{P}_{19.3} \mathrm{I}_{8}$ molecules at the deposition zone can decompose to release $\mathrm{SnI}_{2}, \mathrm{P}_{4}$, and even $\mathrm{P}_{2}$ at the temperature of $\sim 550-500^{\circ} \mathrm{C}$, and thus are highly active. Meanwhile, the remaining $\mathrm{SnI}_{2}$ in the gas phase, functioning as a mineralizer, can transport $\mathrm{P}_{4}$ molecules to the growth sites by forming a Sn-P-I intermediate compound, yielding BP crystals in a polymerization-like process. In addition, we suggest that the SnI assisted synthesis of BP crystals should have the same growth processes, although different $\mathrm{Sn}$ and I containing additives can be used as mineralizers. We believe that this study can improve the understanding of BP synthesis, and facilitate controlled synthesis of $\mathrm{BP}$ for future applications.

\section{EXPERIMENTAL SECTION}

\section{Materials}

Tin powder (Sn, 99.99\%) and iodine granule ( $\left.\mathrm{I}_{2}, \mathrm{AR}, 99.8 \%\right)$ were purchased from Aladdin (Shanghai, China). Red phosphorus (99.999\%, metals basis) was purchased from Alfa Aesar. Acetic acid (AR), acetic anhydride (AR), toluene (AR), and other chemicals were purchased from Shanghai Lingfeng Chemical Reagent Co., Ltd. All chemicals were used as received without further purification unless otherwise noted.

\section{Synthesis of $\mathrm{Snl}_{4}, \mathrm{Snl}_{2}, \mathrm{Sn}_{4} \mathrm{P}_{3}, \mathrm{Sn}_{24} \mathrm{P}_{19.3} \mathrm{I}_{8}$, SnIP, and Black Phosphorus (BP) Synthesis of $\mathrm{Snl}_{4}$ (Köpf et al., 2014)}

To a mixture of acetic acid $(25 \mathrm{~mL})$ and acetic anhydride $(25 \mathrm{~mL}), 0.5 \mathrm{~g} \mathrm{Sn}$ powder and $2 \mathrm{~g} \mathrm{I}_{2}$ were added. The resulting mixture was refluxed at $120^{\circ} \mathrm{C}$ for $90 \mathrm{~min}$, during which the $\mathrm{Sn}$ powder completely disappeared. After the mixture was cooled to room temperature, orange-colored $\mathrm{SnI}_{4}$ crystals appeared and were collected. The obtained $\mathrm{SnI}_{4}$ crystals were recrystallized in chloroform for further use.

\section{Synthesis of $\mathrm{Snl}_{2}$}

Typically, a mixture of $\mathrm{SnI}_{4}(1.2956 \mathrm{~g})$ and $\mathrm{Sn}(246.2 \mathrm{mg})$ powders (molar ratio: 1:1) was loaded in an evacuated silica ampoule ( $\sim 10 \mathrm{~cm}$ in length and $\sim 10 \mathrm{~mm}$ in inner diameter). Subsequently, the ampoule was heated to $400^{\circ} \mathrm{C}$ within $1 \mathrm{~h}$ in a muffle furnace, annealed for $5 \mathrm{~h}$, and cooled down to room temperature naturally. The $\mathrm{SnI}_{2}$ powders, on the wall of the silica ampoule, were collected for further use.

\section{Synthesis of $\mathrm{Sn}_{4} \mathrm{P}_{3}$}

A mixture of grounded red phosphorus powder $(72 \mathrm{mg})$ and tin powder $(364 \mathrm{mg}$ ) was sealed in an evacuated silica ampoule ( $\sim 10 \mathrm{~cm}$ in length and $\sim 10 \mathrm{~mm}$ in inner diameter). The sealed ampoule was then annealed at $400^{\circ} \mathrm{C}$ for $8 \mathrm{~h}$ in a muffle furnace, and cooled to $200^{\circ} \mathrm{C}$ in $2 \mathrm{~h}$. Finally, the ampoule was naturally cooled down to room temperature and the resulting $\mathrm{Sn}_{4} \mathrm{P}_{3}$ was collected.

\section{Synthesis of $\mathrm{Sn}_{24} \mathrm{P}_{19.3} \mathrm{I}_{8}$ and SnIP}

A mixture of tin powder, red phosphorus powder, and $\mathrm{I}_{2}$, with stoichiometric ratio, was sealed in an evacuated silica ampoule. Subsequently, the sealed ampoule was heated to $400^{\circ} \mathrm{C}$ within $40 \mathrm{~min}$, kept for $10 \mathrm{~h}$, and slowly cooled down to room temperature in $75 \mathrm{~h}$. The ternary compounds were then collected from the silica ampoule.

\section{Synthesis of Black Phosphorus (BP)}

In a typical experiment, a mixture of red phosphorus $(300 \mathrm{mg})$ and mineralizers $\left(\mathrm{SnI}_{4}: 6 \mathrm{mg}, \mathrm{Sn}: 12 \mathrm{mg}\right)$ was sealed in an evacuated silica ampoule $(\sim 10 \mathrm{~cm}$ in length and $\sim 10 \mathrm{~mm}$ in inner diameter). The ampoule was placed horizontally in the center zone of a muffle box furnace with a viewing window. It 
should be noted that the temperature at the center of the furnace is slightly different from that near the wall. The temperature of the furnace was increased from room temperature to $650^{\circ} \mathrm{C}$ in $1 \mathrm{~h}$, and then decreased to $550^{\circ} \mathrm{C}$ within $1 \mathrm{~h}$. Afterwards, the temperature was further decreased to $500^{\circ} \mathrm{C}$ in $8 \mathrm{~h}$, and then to $200^{\circ} \mathrm{C}$ in $4 \mathrm{~h}$. Finally, the furnace was turned off for natural cooling to room temperature. After the reaction, BP crystals were collected from the ampoule, washed with hot toluene $\left(\sim 60^{\circ} \mathrm{C}\right)$ for three times until the toluene was colorless, dried at $\mathrm{N}_{2}$ atmosphere and stored at ambient environment.

BP crystals were also prepared in the presence of different mineralizers, such as $\mathrm{Sn}_{24} \mathrm{P}_{19.3} \mathrm{I}_{8}$ (21.6 mg), SnIP (10.6 mg), a mixture of $\mathrm{Sn}$ and $\mathrm{I}_{2}\left(\mathrm{Sn}: 4.5-14 \mathrm{mg}, \mathrm{I}_{2}: 4.8 \mathrm{mg}\right.$ ) and a mixture of $\mathrm{SnI}_{2}$ and $\mathrm{Sn}\left(\mathrm{SnI}_{2}: 7.1 \mathrm{mg}, \mathrm{Sn}: 11.2 \mathrm{mg}\right.$ ), by keeping other conditions identical. Notably, despite the differences among varied mineralizers, the molar amount of I element was kept the same $(0.038 \mathrm{mmol})$ when different mineralizers were used. All synthesis reactions, including the control experiments, were carried out under the same temperature program unless otherwise noted. To record the reaction processes, photographs were taken from the viewing window of the furnace at different stages during the reaction.

\section{CHARACTERIZATION}

Powder X-ray diffraction (XRD) patterns were recorded using a Rigaku Smartlab (9 kW) X-ray diffractometer with $\mathrm{Cu}$ $\mathrm{K} \alpha$ radiation $(\lambda=1.5406 \AA)$. Raman spectra were acquired on a HR Evaluation spectrometer (Horiba, Confocal Raman Microscope) with a $532 \mathrm{~nm}$ laser. Thermogravimetric (TG) analysis was performed on a METTLER TGA2 under the $\mathrm{N}_{2}$ atmosphere at a heating rate of $10^{\circ} \mathrm{C} / \mathrm{min}$. Scanning electron microscopy (SEM) image and corresponding energy dispersive $\mathrm{X}$-Ray spectroscopy (EDX) analysis were carried out in a JSM$7800 \mathrm{~F}$ (JEOL) scanning electron microscope. Low resolution transmission electron microscopy (TEM) measurements were carried out on a JEM-1400 transmission electron microscope at an acceleration voltage of $120 \mathrm{kV}$. High resolution TEM, darkfield scanning transmission electron microscopy (STEM), and corresponding EDX measurements were conducted on a $2100 \mathrm{~F}$ transmission electron microscope at an acceleration voltage of $200 \mathrm{kV}$. Photographs were taken by a digital camera.

\section{RESULTS AND DISCUSSION}

\section{Characterization of BP Crystals}

Prior to studying the growth process of BP crystals, we first confirmed that high quality BP crystals can be obtained under our synthetic conditions. On a separate note, considering that the mixture of $\mathrm{SnI}_{4}$ and $\mathrm{Sn}$ is one of the most commonly used mineralizers, we here chose the $\mathrm{SnI}_{4} / \mathrm{Sn}$ mixture as a representative mineralizer in the following sets of experiments. As shown in Figure 1A, the obtained bulk BP crystals present a flower-like (radial) shape, with many sheetlike branches, on a centimeter scale and black color with metallic luster. Meanwhile, as revealed by the scanning electron microscopy (SEM) image, the BP crystal has a layered structure (Figure 1B), exhibiting the essential feature as a layered material (Figure 1C, upper panel). Further powder X-ray diffraction (XRD) analysis shows that the as-synthesized BP crystals give three main diffraction peaks at $\sim 16,34$, and $52^{\circ}$ (Figure 1D). The XRD pattern reveals that the BP crystals are in orthorhombic phase with excellent crystallinity, while the three diffraction peaks indicate that the crystals grow in a highly oriented manner. Consistently, in the Raman spectrum, three characteristic peaks, at $\sim 361,438$, and $466 \mathrm{~cm}^{-1}$, were observed (Figure 1E), which can be attributed to the characteristic $A_{\mathrm{g}}^{1}, \mathrm{~B}_{2 \mathrm{~g}}$, and $\mathrm{A}_{\mathrm{g}}^{2}$ modes in the orthorhombic BP lattice (Figure 1C, lower panel) (Guo et al., 2015; Ribeiro et al., 2018; Wang et al., 2018). In addition, high resolution transmission electron microscopy analysis of the exfoliated BP sheets, together with the energy dispersive X-Ray spectroscopy (EDX) analysis, shows the good crystallinity and purity of the assynthesized BP crystals (Figure S1). Collectively, orthorhombic BP crystals, with excellent quality, can be successfully synthesized in the presence of the $\mathrm{SnI}_{4} / \mathrm{Sn}$ mineralizer under our reaction conditions.

\section{Influence of Mineralizer Composition on the Synthesis of BP Crystals}

In order to understand the functions of the Sn-I containing mineralizers in the reactions, we first systematically tuned the molar ratio of $\mathrm{Sn}$ and I elements in the mineralizer by changing the amount of Sn. We found that the molar ratio of Sn:I had a threshold at $\sim 0.9: 1$, below which almost no BP crystals can be synthesized (Figure 2A and Figure S2). It should be mentioned that a similar threshold of the Sn:I molar ratio (2:1) was also observed by a previous report ( $\mathrm{Li}$ et al., 2017), although the reported ratio is slightly different from that we found here. The difference may be due to variations in reaction conditions, including heating process, silica tube, and furnace. In particular, BP crystals with high quality can be obtained in high yield ( $\sim 90 \%$ ) when the molar ratio of Sn:I is in the range from $\sim 0.9: 1$ to 3:1 (Figures 2A,B). Further increase of the Sn:I ratio can also produce BP crystals, but the yield of BP crystals declines in most cases. For example, when the Sn:I ratio was set at $\sim 10: 1$, smallsized BP crystals, scattered on the inner wall of the ampoule, were observed with a large number of side products (Figure 2B). These results indicate that the optimal molar element ratio of Sn and I, for preparing high quality BP crystals in high yield under our conditions, is in the range from $\sim 0.9: 1$ to $3: 1$, when the $\mathrm{SnI}_{4} / \mathrm{Sn}$ mineralizer is used.

To check whether the Sn:I ratio for BP synthesis is dependent on the types of the Sn-I containing mineralizers, we then employed other commonly used Sn-I containing mineralizers, such as $\mathrm{Sn} / \mathrm{I}_{2}$ and $\mathrm{SnI}_{2} / \mathrm{Sn}$ (Figure S3), to synthesize BP crystals under the same reaction conditions. As expected, high quality BP crystals can be successfully prepared in the presence of $\mathrm{Sn} / \mathrm{I}_{2}$ or $\mathrm{SnI}_{2} / \mathrm{Sn}$ mineralizer if the $\mathrm{Sn}$ :I ratio was kept in the range from $\sim 0.9: 1$ to 3:1 (Figure 2B and Figure S4). If the Sn:I ratio was set smaller than $\sim 0.9: 1$, the reproducibility of the reaction became poor and few BP crystals were obtained in most cases (Figure S5). To further verify the Sn:I ratio, two ternary Sn-P-I compounds, SnIP (Figure S6) and $\mathrm{Sn}_{24} \mathrm{P}_{19.3} \mathrm{I}_{8}$ (Figure S7), were 
A

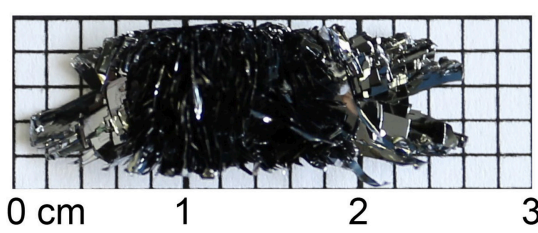

B

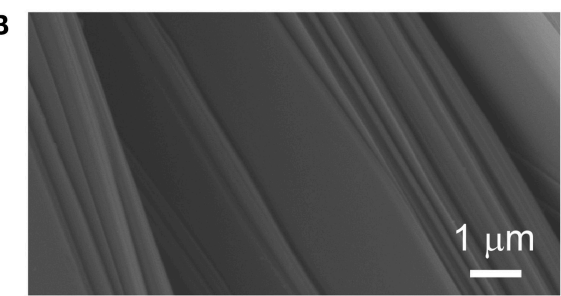

D

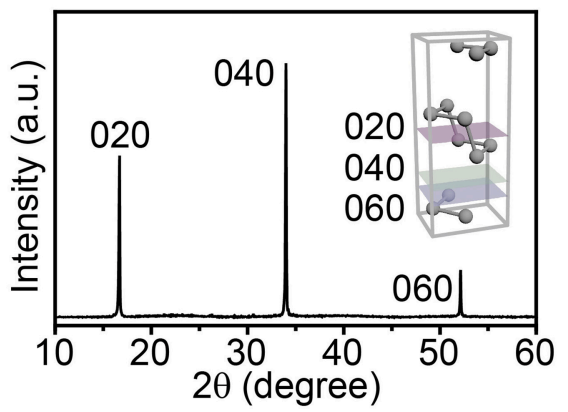

C

$5.3 \AA$

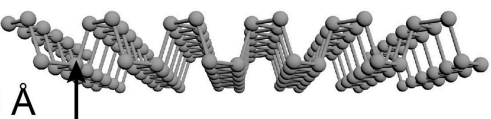

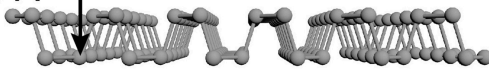
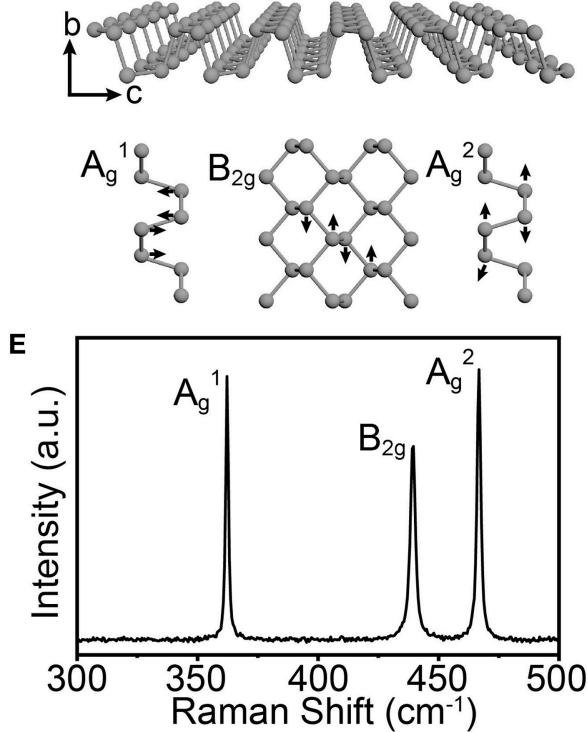

FIGURE 1 | (A) Photo of the as-prepared bulk BP crystals. (B) SEM image showing the layered structure of the BP crystal. (C) Schematic illustrations of the atomic structure of orthorhombic BP and corresponding vibrational modes in Raman spectroscopy. (D) Powder XRD pattern of the obtained BP crystals. Inset: schematic illustration of a crystal cell of orthorhombic BP and its crystal planes. (E) Raman spectrum of the as-prepared BP.

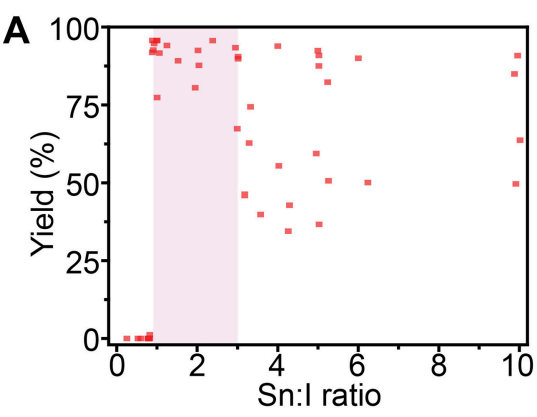

B

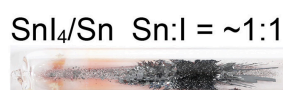

$S n: I=\sim 10: 1$
$\mathrm{Sn} / \mathrm{I}_{2} \quad \mathrm{Sn}: \mathrm{I}=\sim 1: 1$

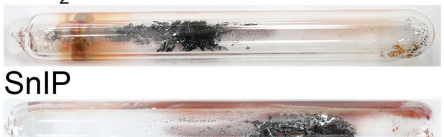

$\mathrm{Sn}_{24} \mathrm{P}_{19.3} \mathrm{I}_{8}$

FIGURE 2 | (A) Distribution of the synthetic yield of BP crystals against Sn:I ratio in the Snl 4 /Sn assisted reaction. (B) Photos of BP crystals in ampoules obtained in the presence of different mineralizers.

applied as mineralizers (Shatruk et al., 1999; Pfister et al., 2016). It should be mentioned that the Sn:I ratio of each Sn-P-I compound is near either the upper or lower limit of the determined range. Similar to other mineralizers, both $\mathrm{SnIP}$ and $\mathrm{Sn}_{24} \mathrm{P}_{19.3} \mathrm{I}_{8}$ can yield centimeter-sized high quality BP crystals (Figure 2B) under the same reaction conditions, validating the determined optimum range of $\mathrm{Sn}$ :I ratio.

Collectively, these results reveal that the mineralizers, despite the different chemical compositions such as $\mathrm{SnI}_{4} / \mathrm{Sn}, \mathrm{Sn} / \mathrm{I}_{2}$, $\mathrm{SnI}_{2} / \mathrm{Sn}$, SnIP, and $\mathrm{Sn}_{24} \mathrm{P}_{19.3} \mathrm{I}_{8}$, can facilitate the growth of BP crystals if a proper molar ratio of $\mathrm{Sn}$ and I elements is applied. Furthermore, according to these results, we can suppose that all
Sn-I containing mineralizers should facilitate the formation of BP crystals through the same way.

\section{Growth of BP Crystals and Transformation of Mineralizer}

Next, we monitored the synthesis of BP crystals (Figure 3 and Figure S8), by taking in situ photos at different stages during the reaction as marked in Figure $\mathbf{3 A}$, to reveal the functions of the mineralizer. For comparison, pure red phosphorus and the $\mathrm{SnI}_{4} / \mathrm{Sn}$ mineralizer were also sealed solely in ampoules and monitored under the same conditions. As shown in Figure 3B1, the solid reactants initially were placed at the right side of 
the sealed ampoules. With the increase of the temperature, the $\mathrm{SnI}_{4} / \mathrm{Sn}$ mineralizer began to react with red phosphorus, exhibiting some mineralization effects at $\sim 350^{\circ} \mathrm{C}$ (Figure 3B2, dotted box). When the temperature reached $650^{\circ} \mathrm{C}$, almost all reactants including the mineralizer became vapor, leaving small amount of reactants at the original zone, and the whole ampoule exhibited orange color (Figure 3B3). Notably, the same orange color was also observed in the ampoule containing only the $\mathrm{SnI}_{4} / \mathrm{Sn}$ mineralizer, indicating the orange color comes from the vapor of mineralizer. Finally, the BP crystals appeared at the other side of the ampoule during the decrease of temperature, while some solid remained at the zone where the initial reactants were placed (Figures 3B5,B6). Interestingly, in the ampoules containing only the $\mathrm{SnI}_{4} / \mathrm{Sn}$ mineralizer, some orangered colored solid appeared at almost the same position where BP crystals grew (Figure 3B6, dotted boxes). In stark contrast, in the absence of the mineralizer, the red phosphorus finally deposited randomly in the ampoules. Furthermore, we observed similar growth processes, with slight differences, when different types of mineralizers, such as $\mathrm{Sn} / \mathrm{I}_{2}, \mathrm{SnIP}$, and $\mathrm{Sn}_{24} \mathrm{P}_{19.3} \mathrm{I}_{8}$, were used (Figures S9-S11). These results show that the Sn-I containing mineralizer may first undergo transformation to form certain compounds and then react with phosphorus vapor to help the formation of BP crystals during the reaction.

To gain more insights into the growth process of BP crystals, we then tried to identify the transformation of mineralizer during the reaction. According to the thermogravimetric (TG) analysis (Figure 3C) and in situ photos (Figure 3B), all reactants, except $\mathrm{Sn}$, should become vapor or decompose at $650^{\circ} \mathrm{C}$. Comparing the status of pure $\mathrm{I}_{2}, \mathrm{SnI}_{4}$, and $\mathrm{SnI}_{2}$ at $650^{\circ} \mathrm{C}$ (Figure $\mathrm{S12}$ ) with the observation shown in Figure $\mathbf{3 B}$, we can deduce that the mineralizer should decompose to form gaseous $\mathrm{SnI}_{2}$ that exhibits orange color during the reaction. This deduction is also consistent with the previous report in which $\mathrm{SnI}_{2}$ is believed to be the most thermodynamically stable species in the reaction system at $650^{\circ} \mathrm{C}$ ( $\mathrm{Li}$ et al., 2017). Regarding the material remaining at the zone where the initial reactants are placed at $650^{\circ} \mathrm{C}$, we believe that it is liquid $\mathrm{Sn}$ because the boiling point of $\mathrm{Sn}$ is $\sim 2,600^{\circ} \mathrm{C}$ (Dean, 1999). In addition, other compounds, including the common Sn-P and Sn-P-I compounds, cannot remain intact at such high temperature $\left(\sim 650^{\circ} \mathrm{C}\right)$ according to the TG analysis (Figure 3C and Figure S13). Accordingly, in the Sn-I assisted synthesis of BP crystals, we suggest that the SnI containing mineralizer should form $\mathrm{SnI}_{2}$ and $\mathrm{Sn}$ during the reaction.

To further verify the transformation of the mineralizer, we heated the SnIP and $\mathrm{Sn}_{24} \mathrm{P}_{19.3} \mathrm{I}_{8}$ compounds under the same reaction conditions and then analyzed the final compounds (Figure S14). As expected, $\mathrm{SnI}_{2}$ was observed at the place away from the zone where initial compounds were placed. Meanwhile, $\mathrm{Sn}_{4} \mathrm{P}_{3}$, formed by the reaction of $\mathrm{Sn}$ and $\mathrm{P}$ during the cooling process, appeared at the zone which originally contains the Sn-I$\mathrm{P}$ compounds. In addition, we also found the presence of $\mathrm{SnI}_{2}$ on the as-prepared BP crystals. In this set of experiments, the BP crystals, prepared with the assistance of various mineralizers, were collected from the ampoule and then immersed into hot toluene. The toluene, with light yellow color (Figure S15), was then distilled, producing a few powders. As characterized by Raman spectroscopy, the powders can be identified as $\mathrm{SnI}_{2}$ (Figure S15).

Taken together, in the Sn-I assisted synthesis of BP crystals, the Sn-I containing mineralizer should first form sufficient $\mathrm{SnI}_{2}$. Subsequently, the gaseous $\mathrm{SnI}_{2}$ can react with gaseous phosphorus, forming a certain active Sn-P-I intermediate, and assist the formation of BP crystals.

\section{Active Site Identification and Characterization}

In the following set of experiments, we intended to figure out the gaseous Sn-P-I intermediate and growth details of BP crystals by both monitoring the reaction and characterizing the assynthesized BP crystals. Actually, we observed that BP crystals grew from the bottom of the ampoule at the cooling stage (mainly from 550 to $500^{\circ} \mathrm{C}$ ) in reactions (Figure 3 and Figures S8-S11), and the growth of the crystals apparently exhibited an epitaxial manner. After the reaction, we collected the BP crystals from the ampoule tube and checked their back side which formerly attached on the wall of the ampoule. As shown in Figures 4A,B, a distinct intersection point with clear trails can be typically observed, indicating that the point is the starting point for growing BP crystals. Thus, we here suggest that the intersection point should be the active site for the growth of BP crystals. It should be mentioned that similar intersection points were also observed and considered as the nucleation sites for BP growth in previous studies (Zhao et al., 2016b).

Consequently, we analyzed the composition of the intersection point by energy dispersive X-Ray spectroscopy (EDX). At the intersection point of the as-prepared BP crystal, the molar ratio of $\mathrm{Sn}: \mathrm{I}$ was determined as $\sim 0.6: 1$ by EDX (Figure S16). After thoroughly washed by hot toluene, as exampled in Figures 4C-F, the Sn:I ratio at the intersection point was found as $\sim 3.1: 1$ (Figure S17). Similarly, for the BP crystals obtained in the presence of $\mathrm{Sn}_{24} \mathrm{P}_{19.3} \mathrm{I}_{8}$ as a mineralizer, the $S n: I$ ratio at the intersection point was found as $\sim 2.9: 1$ after toluene washing (Figure S18). Taking previous studies into account (Zhang et al., 2016; Li et al., 2017), we believe that the $\mathrm{Sn}_{24} \mathrm{P}_{19.3} \mathrm{I}_{8}$ compound should serve as the active site for the growth of BP crystals.

Besides, in order to find out if there were any other components at the active site, we studied the intersection point of BP by Raman spectroscopy. By comparing the obtained Raman spectra at the intersection point, the presence of Hittorf's phosphorus can be deduced (Figure S19). It should be pointed out that we also checked the products just near the intersection point by Raman spectroscopy. Only characteristic Raman peaks of BP crystals were observed (Figure S19). These results show that Hittorf's phosphorus may also play a certain role during the growth of BP crystals.

\section{Growth Mechanism of BP Crystals}

As discussed in the former parts, in the Sn-I assisted synthesis of $\mathrm{BP}$ crystals, $\mathrm{SnI}_{2}$ should be a critical gaseous compound that may form gaseous intermediate to facilitate the growth of BP. Meanwhile, $\mathrm{Sn}_{24} \mathrm{P}_{19.3} \mathrm{I}_{8}$ should be the active site on 

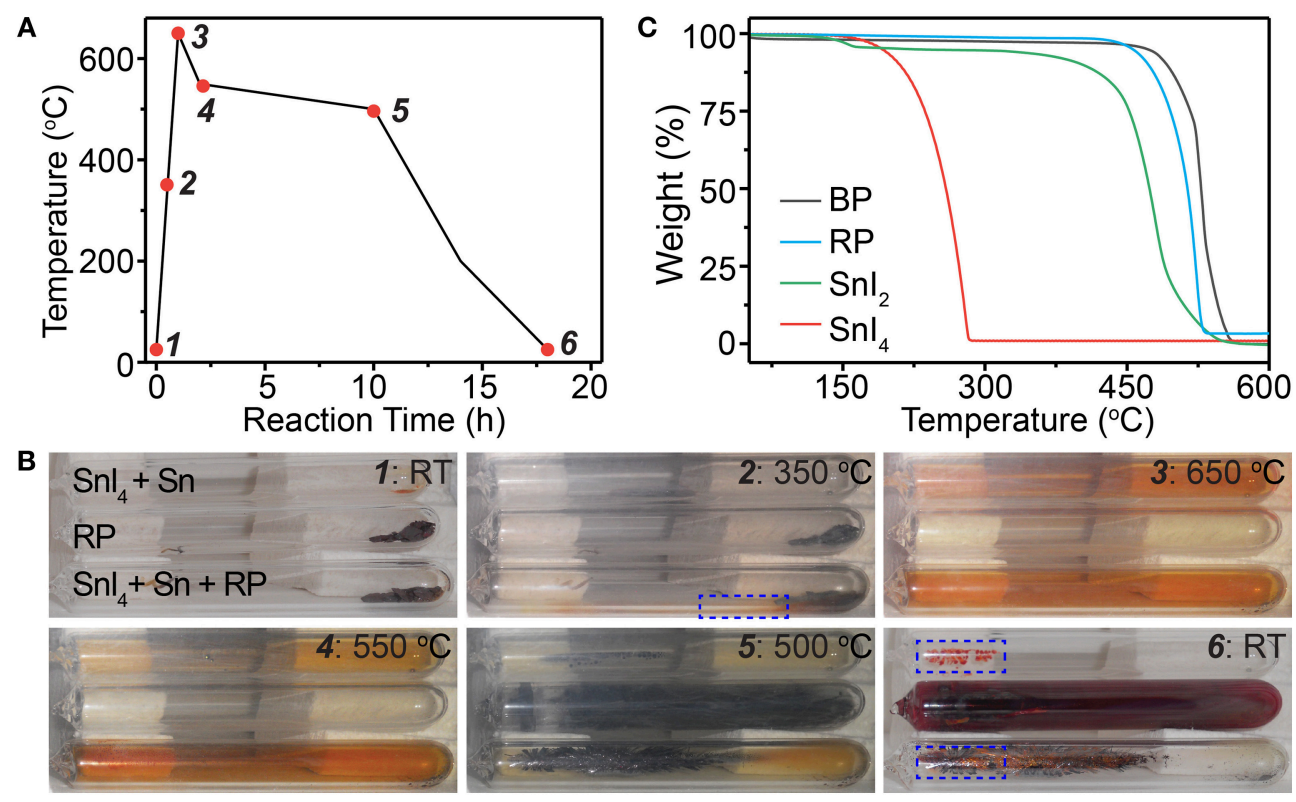

FIGURE 3 | (A) Typical time dependent temperature profile for synthesizing BP crystals and (B) corresponding in situ photos taken during the synthesis. The red dots in the profile indicate the stages when the photos are taken. (C) Thermogravimetric profiles of different compounds. Note that RT and RP denote room temperature and red phosphorus, respectively.
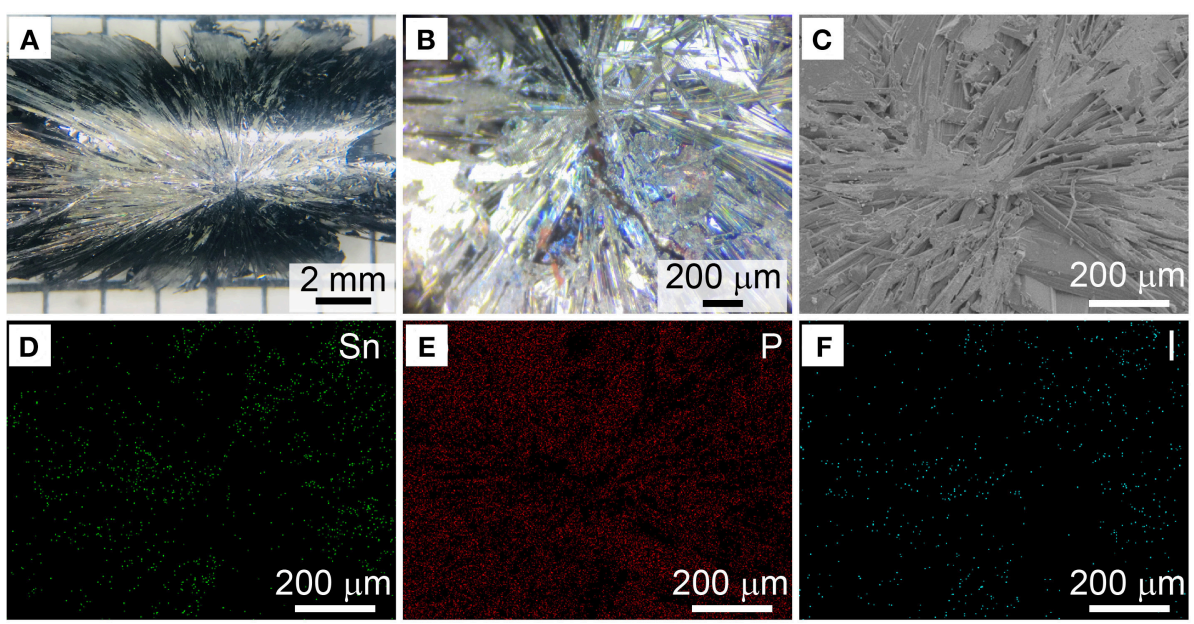

FIGURE 4 | (A) Representative photo of the as-obtained BP crystals with an intersection point. (B) Corresponding enlarged photo of the intersection point. (C) SEM image of a randomly selected intersection point. (D-F) Corresponding elemental mapping of the intersection point shown in (C).

which the BP crystals start to grow. Combining our results, the fundamentals of chemical vapor transport reactions (Binnewies et al., 2012), and previous proposed growth mechanisms of BP crystals together (Zhao et al., 2016a,b; Li et al., 2017; Zhang Z. et al., 2017; Shriber et al., 2018), we here propose the following formation mechanism of BP crystals in the Sn-I assisted reaction (Figure 5A).

At the beginning of the Sn-I assisted reaction, temperature increases for reactant sublimation. At this stage, despite different mineralizers, all reactants, placed at the right side of the ampoule in our studies (Figure 3), sublime or decompose gradually to generate gaseous $\mathrm{SnI}_{2}$, gaseous $\mathrm{P}_{4}$, and liquid $\mathrm{Sn}$. The resulting gaseous $\mathrm{SnI}_{2}$, gaseous $\mathrm{P}_{4}$, and liquid $\mathrm{Sn}$ can further react to form $\mathrm{Sn}_{24} \mathrm{P}_{19.3} \mathrm{I}_{8}$ (Equation 1) and also probably some other Sn-P-I compounds at high temperature (e.g., $\sim 650^{\circ} \mathrm{C}$ in this study).

$$
4 \mathrm{SnI}_{2}+20 \mathrm{Sn}+4.825 \mathrm{P}_{4} \leftrightarrow \mathrm{Sn}_{24} \mathrm{P}_{19.3} \mathrm{I}_{8}
$$

Notably, in a sealed ampoule, the reaction (Equation 1), at high temperature, can be at equilibrium that is neither reactantfavored nor product-favored. Moreover, synthesis of $\mathrm{Sn}_{24} \mathrm{P}_{19.3} \mathrm{I}_{8}$ 
A

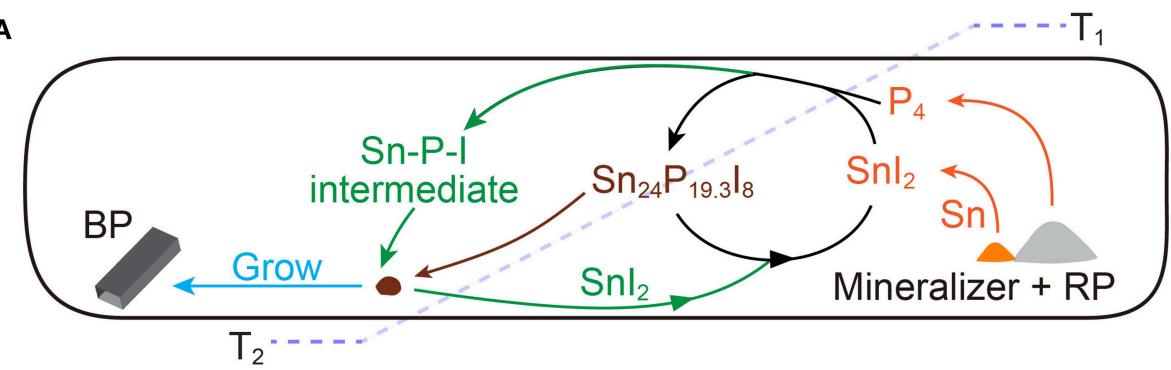

B

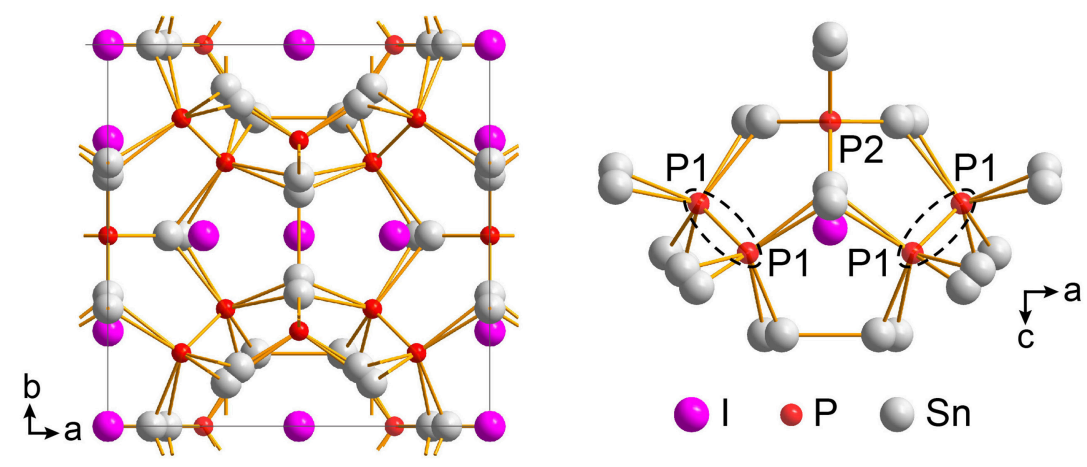

FIGURE 5 | (A) Schematic illustration of the proposed mechanism of BP growth in the Sn-I assisted reaction. The mineralizer and red phosphorus (RP) first form Snl 2 , $\mathrm{P}_{4}$, and $\mathrm{Sn}$ at high temperature $\left(\mathrm{T}_{1}\right)$ (orange colored arrows). The resulting $\mathrm{Snl}_{2}, \mathrm{P}_{4}$, and $\mathrm{Sn}$ then react to yield $\mathrm{Sn}_{24} \mathrm{P}_{19.3} \mathrm{l}_{8}$ which can deposit at the place with relatively low temperature $\left(\mathrm{T}_{2}\right)$ (brown colored arrow). Subsequently, the BP crystals can grow with the assistance of $\mathrm{Snl} 2$ (green colored arrows). (B) Crystal structure of $\mathrm{Sn}_{24} \mathrm{P}_{19.3} \mathrm{l}_{8}$ where $\mathrm{Sn}$ and $\mathrm{P}$ atoms form a three dimensional framework with I atoms as guests.

typically needs much longer time than that for preparing BP crystals (Shatruk et al., 1999). Therefore, we suggest that there are still some gaseous $\mathrm{SnI}_{2}$ in the reaction system. Regarding the liquid $\mathrm{Sn}$ presented at this stage, we believe that the slightly excess $\mathrm{Sn}$ can ensure the formation of $\mathrm{Sn}_{24} \mathrm{P}_{19.3} \mathrm{I}_{8}$ and gaseous $\mathrm{SnI}_{2}$.

After the temperature of the reaction system reaches $650^{\circ} \mathrm{C}$, the reaction temperature starts to cool to $500^{\circ} \mathrm{C}$ with a low cooling rate. At this cooling stage, some $\mathrm{Sn}_{24} \mathrm{P}_{19.3} \mathrm{I}_{8}$ molecules deposit first at the place with a bit lower temperature because of vapor oversaturation. Remarkably, we here propose that $\mathrm{Sn}_{24} \mathrm{P}_{19.3} \mathrm{I}_{8}$, at the temperature of $\sim 550-500^{\circ} \mathrm{C}$, should be highly active as growth sites, due to its unique structure. Structurally, $\mathrm{Sn}_{24} \mathrm{P}_{19.3} \mathrm{I}_{8}$, with a clathrate type-I structure, is a three dimensional framework of tin and phosphorus atoms (Figure 5B, left panel), where I atoms are guests in the framework (Shatruk et al., 1999). There are two types of $\mathrm{P}$ atoms in the structure, and one type of $\mathrm{P}$ atoms appears in pairs with a P-P separation of $2.20 \AA$, similar to typical P-P bond (Figure 5B, right panel) (Shatruk et al., 1999). Furthermore, $\mathrm{Sn}_{24} \mathrm{P}_{19.3} \mathrm{I}_{8}$ crystals typically can have phosphorus vacancies in their structure ( $\mathrm{Li}$ et al., 2017). Taking the TG analysis into account (Figure S13), we thus reason that the positions for $\mathrm{P}$ atoms in the $\mathrm{Sn}_{24} \mathrm{P}_{19.3} \mathrm{I}_{8}$ crystals, either $\mathrm{P}$ atoms or vacancies, can be the starting points for $\mathrm{BP}$ growth at the temperature of $\sim 550-500^{\circ} \mathrm{C}$.

After the formation of growth sites, BP crystals then can continuously grow with the assistance of $\mathrm{SnI}_{2}$, following a typical chemical vapor transportation process. More specifically, gaseous $\mathrm{SnI}_{2}$ molecules first react with gaseous $\mathrm{P}_{4}$ molecules at the high temperature zone, forming a Sn-P-I intermediate. The intermediate compounds then migrate to the zone with slightly lower temperature, where the growth sites are formerly formed. Subsequently, the intermediate compounds react with the active $\mathrm{Sn}_{24} \mathrm{P}_{19.3} \mathrm{I}_{8}$, yielding $\mathrm{BP}$ and releasing $\mathrm{SnI}_{2}$ for further reaction. The reaction would continue like polymerization until most of the $\mathrm{P}_{4}$ molecules are consumed. Finally, the gaseous $\mathrm{SnI}_{2}$ molecules are deposited randomly, mainly in the zone with lower temperature, when the temperature further decreases.

On a separate note, at the intersection points (growth sites) of the obtained BP crystals, we found Hittorf's phosphorus (Figure S19) which was also observed in some former studies (Chen et al., 2017; Zhang Z. et al., 2017). According to the experimental observations, Hittorf's phosphorus may function as the active sites or intermediate state for BP growth (Chen et al., 2017; Zhang Z. et al., 2017). However, considering that Hittorf's phosphorus can be directly obtained by heating red phosphorus in a vacuum container (Chen et al., 2017), we here believe that Hittorf's phosphorus should be the by-product formed at the beginning of BP growth.

\section{CONCLUSION}

In conclusion, we have successfully synthesized BP crystals with various mineralizers, including $\mathrm{SnI}_{4} / \mathrm{Sn}, \mathrm{SnI}_{2} / \mathrm{Sn}, \mathrm{Sn} / \mathrm{I}_{2}$, SnIP, and $\mathrm{Sn}_{24} \mathrm{P}_{19.3} \mathrm{I}_{8}$. By monitoring the $\mathrm{Sn}-\mathrm{I}$ assisted reactions and characterizing the products, together with a series of control experiments, we here propose that BP crystals grow 
through a $\mathrm{SnI}_{2}$ mineralized reaction, and the $\mathrm{Sn}_{24} \mathrm{P}_{19.3} \mathrm{I}_{8}$ compound serves as an active site for BP growth. Although more detailed in situ studies are still needed, we believe that our studies shown here should provide insights into the growth mechanism of BP crystals, facilitating further manipulation of BP synthesis.

\section{AUTHOR CONTRIBUTIONS}

ML and XX conceived and designed the experiments. DW, PY, and LZ performed experiments. LW and HL contributed in the Raman analysis. ML, XX, LH, and WH supervised the research and contributed to the manuscript writing. All authors read and approved the final manuscript.

\section{REFERENCES}

Binnewies, M., Glaum, R., Schmidt, M., and Schmidt, P. (2012). Chemical Vapor Transport Reactions. Berlin; Boston, BA: Walter de Gruyter GmbH. \& Co. KG.

Bridgman, P. W. (1914). Two new modifications of phosphorus. J. Am. Chem. Soc. 36, 1344-1363. doi: 10.1021/ja02184a002

Bridgman, P. W. (1935). Effects of high shearing stress combined with high hydrostatic pressure. Phys. Rev. 48, 825-847. doi: 10.1103/PhysRev. 48.825

Bridgman, P. W. (1948). The compression of 39 substances to $100,000 \mathrm{~kg} / \mathrm{cm}^{2}$. Proc. Am. Acad. Arts Sci. 76, 55-70.

Chen, Z., Zhu, Y., Lei, J., Liu, W., Xu, Y., and Feng, P. (2017). A stage-by-stage phase-induction and nucleation of black phosphorus from red phosphorus under low-pressure mineralization. CrystEngComm 19, 7207-7212. doi: 10.1039/C7CE01492A

Dean, J. A. (1999). Lange's Handbook of Chemistry, 15th edn. New York, NY: McGraw-Hill Inc.

Guo, Z., Zhang, H., Lu, S., Wang, Z., Tang, S., Shao, J., et al. (2015). From black phosphorus to phosphorene: basic solvent exfoliation, evolution of Raman scattering, and applications to ultrafast photonics. Adv. Funct. Mater. 25, 6996-7002. doi: 10.1002/adfm.201 502902

Hirsch, A., and Hauke, F. (2018). Post-graphene 2D. chemistry: the emerging field of molybdenum disulfide and black phosphorus functionalization. Angew. Chem. Int. Ed. Engl. 57, 4338-4354. doi: 10.1002/anie.2017 08211

Hu, Z., Niu, T., Guo, R., Zhang, J., Lai, M., He, J., et al. (2018). Two-dimensional black phosphorus: its fabrication, functionalization and applications. Nanoscale 10, 21575-21603. doi: 10.1039/c8nr07395c

Köpf, M., Eckstein, N., Pfister, D., Grotz, C., Krüger, I., Greiwe, M., et al. (2014). Access and in situ growth of phosphorene-precursor black phosphorus. J. Cryst. Growth 405, 6-10. doi: 10.1016/j.jcrysgro.2014.07.029

Lange, S., Schmidt, P., and Nilges, T. (2007). Au $\mathrm{An}_{3} \mathrm{P}_{7} @$ black phosphorus: an easy access to black phosphorus. Inorg. Chem. 46, 4028-4035. doi: 10.1021/ic062192q

Li, L., Yu, Y., Ye, G. J., Ge, Q., Ou, X., Wu, H., et al. (2014). Black phosphorus field-effect transistors. Nat. Nanotech. 9, 372-377. doi: 10.1038/nnano. 2014.35

Li, S., Liu, X., Fan, X., Ni, Y., Miracle, J., Theodoropoulou, N., et al. (2017). New strategy for black phosphorus crystal growth through ternary clathrate. Cryst. Growth Des. 17, 6579-6585. doi: 10.1021/acs.cgd.7b 01239

Liu, H., Du, Y., Deng, Y., and Ye, P. D. (2015). Semiconducting black phosphorus: synthesis, transport properties and electronic applications. Chem. Soc. Rev. 44, 2732-2743. doi: 10.1039/C4CS00257A

\section{FUNDING}

This work was supported by National Key R\&D Program of China (2017YFA0207201), National Natural Science Foundation of China (21507059), Young Elite Scientists Sponsorship Program by CAST (2017QNRC001), Six Talent Peaks Project in Jiangsu Province (JNHB-038), and Scientific Research Foundation for the Returned Overseas Chinese Scholars, Ministry of Education.

\section{SUPPLEMENTARY MATERIAL}

The Supplementary Material for this article can be found online at: https://www.frontiersin.org/articles/10.3389/fchem. 2019.00021/full\#supplementary-material

Liu, H., Hu, K., Yan, D., Chen, R., Zou, Y., Liu, H., et al. (2018). Recent advances on black phosphorus for energy storage, catalysis, and sensor applications. Adv. Mater. 30:1800295. doi: 10.1002/adma.2018 00295

Liu, Y., Duan, X., Huang, Y., and Duan, X. (2018). Two-dimensional transistors beyond graphene and TMDCs. Chem. Soc. Rev. 47, 6388-6409. doi: 10.1039/C8CS00318A

Nilges, T., Kersting, M., and Pfeifer, T. (2008). A fast low-pressure transport route to large black phosphorus single crystals. J. Solid State Chem. 181, 1707-1711. doi: 10.1016/j.jssc.2008.03.008

Pfister, D., Schäfer, K., Ott, C., Gerke, B., Pöttgen, R., Janka, O., et al. (2016). Inorganic double helices in semiconducting SnIP. Adv. Mater. 28, 9783-9791. doi: 10.1002/adma.201603135

Ribeiro, H. B., Pimenta, M. A., and de Matos, C. J. S. (2018). Raman spectroscopy in black phosphorus. J. Raman Spectrosc. 49, 76-90. doi: 10.1002/jr s.5238

Shatruk, M. M., Kovnir, K. A., Shevelkov, A. V., Presniakov, I. A., and Popovkin, B. A. (1999). First tin pnictide halides $\mathrm{Sn}_{24} \mathrm{P}_{19 \cdot 3} \mathrm{I}_{8}$ and $\mathrm{Sn}_{24} \mathrm{As}_{19 \cdot 3} \mathrm{I}_{8}$ : synthesis and the clathrate-I type of the crystal structure. Inorg. Chem. 38, 3455-3457. doi: 10.1021/ic990153r

Shriber, P., Samanta, A., Nessim, G. D., and Grinberg, I. (2018). First-principles investigation of black phosphorus synthesis. J. Phys. Chem. Lett. 9, 1759-1764. doi: 10.1021/acs.jpclett.8b00055

Smith, J. B., Hagaman, D., and Ji, H. F. (2016). Growth of 2D. black phosphorus film from chemical vapor deposition. Nanotechnology 27:215602. doi: 10.1088/0957-4484/27/21/215602

Wang, C., He, Q., Halim, U., Liu, Y., Zhu, E., Lin, Z., et al. (2018). Monolayer atomic crystal molecular superlattices. Nature 555, 231-236. doi: $10.1038 /$ nature25774

Xia, F., Wang, H., Xiao, D., Dubey, M., and Ramasubramaniam, A. (2014). Two-dimensional material nanophotonics. Nat. Photon. 8, 899-907. doi: 10.1038/nphoton.2014.271

Yang, Z., Hao, J., Yuan, S., Lin, S., Yau, H. M., Dai, J., et al. (2015). Fieldeffect transistors based on amorphous black phosphorus ultrathin films by pulsed laser deposition. Adv. Mater. 27, 3748-3754. doi: 10.1002/adma.2015 00990

Zhang, J. L., Han, C., Hu, Z., Wang, L., Liu, L., Wee, A. T. S., et al. (2018). 2D phosphorene: epitaxial growth and interface engineering for electronic devices. Adv. Mater. 30:1802207. doi: 10.1002/adma.2018 02207

Zhang, Y., Zheng, Y., Rui, K., Hng, H. H., Hippalgaonkar, K., Xu, J., et al. (2017). 2D black phosphorus for energy storage and thermoelectric applications. Small 13:1700661. doi: 10.1002/smll.2017 00661

Zhang, Z., Xin, X., Yan, Q., Li, Q., Yang, Y., and Ren, T. L. (2016). Two-step heating synthesis of sub,-3 millimeter-sized orthorhombic black phosphorus 
single crystal by chemical vapor transport reaction method. Sci. China Mater. 59, 122-134. doi: 10.1007/s40843-016-0122-1

Zhang, Z., Xing, D. H., Li, J., and Yan, Q. (2017). Hittorf's phosphorus: the missing link during transformation of red phosphorus to black phosphorus. CrystEngComm 19, 905-909. doi: 10.1039/C6CE02550A

Zhao, M., Niu, X., Guan, L., Qian, H., Wang, W., Sha, J., et al. (2016a). Understanding the growth of black phosphorus crystals. CrystEngComm 18, 7737-7744. doi: 10.1039/C6CE01608A

Zhao, M., Qian, H., Niu, X., Wang, W., Guan, L., Sha, J., et al. (2016b). Growth mechanism and enhanced yield of black phosphorus microribbons. Cryst. Growth Des. 16, 1096-1103. doi: 10.1021/acs.cgd.5b01709
Conflict of Interest Statement: The authors declare that the research was conducted in the absence of any commercial or financial relationships that could be construed as a potential conflict of interest.

Copyright $\odot 2019$ Wang, Yi, Wang, Zhang, Li, Lu, Xie, Huang and Huang. This is an open-access article distributed under the terms of the Creative Commons Attribution License (CC BY). The use, distribution or reproduction in other forums is permitted, provided the original author(s) and the copyright owner(s) are credited and that the original publication in this journal is cited, in accordance with accepted academic practice. No use, distribution or reproduction is permitted which does not comply with these terms. 